\title{
Human Brain Anatomical Connectivity Analysis Using Sequential Sampling and Resampling
}

\author{
Bo Zheng ${ }^{1}$ and Jagath C. Rajapakse ${ }^{1,2}$ \\ ${ }^{1}$ BioInformatics Research Center, School of Computer Engineering, \\ Nanyang Technological University, 50 Nanyang Avenue, \\ Singapore 639798 \\ 2 Singapore-MIT Alliance, N2-B2C-15, 50 Nanyang Avenue, Singapore
}

\begin{abstract}
Diffusion Tensor MR Imaging (DTI) provides non-invasive approach to track white matter (WM) trajectories within human brain in vivo, and thereby facilitates studies of anatomical connectivity between sub-cortical and cortical regions. This paper presents a probabilistic fiber tracking framework, which aims to address the two problems in earlier approaches: first, it does not adopt fractional anisotropy (FA) as the stopping criteria so that the exploration of cortico-cortical connectivity is feasible; secondly, fiber tracking process is regularized so that trajectory with low curvature means high belief of connection between two voxels.
\end{abstract}

\section{Introduction}

Diffusion tensor MR Imaging (DTI) fiber tracking algorithms provide potential for non-invasive reconstructing of white matter (WM) trajectories of human brain in vivo, as well as assessing changes due to disease, such as multiple sclerosis, amyotrophic lateral sclerosis (ALS), stroke, schizophrenia, reading disability, etc [1. In early work, one of the most popular approaches is a simple deterministic line propagation technique whereby a single trajectory is propagated bidirectionally from a manually defined seed point by moving in a direction parallel to principle diffusion direction (PDD) 2345667. However, there are three major limitations to such a deterministic approach. First, they assume that PDD is the tangent vector of underlying dominant WM trajectory orientation in each voxel and estimated from measured DTI data that is discrete, coarsely sampled noisy, and voxel averaged. Hence, there is uncertainty caused by the noise and artifacts present in any MR scan and incomplete modeling of diffusion signal [8] associated with PDD. Furthermore, the reliability of reconstructed WM trajectory cannot be estimated using such deterministic approaches. Secondly, they cannot handle a voxel which contains more than one WM trajectories with different orientations since only one trajectory within each voxel is presumed by single tensor model. Especially, at millimeter-scale resolution of DTI the number of such voxels may be considerate given widespread divergence and convergence of WM trajectories [9]10. And such voxels manifest by the form of oblate diffusion tensor (pancakelike shape) [1]. By examining DTI data of single subject, about $9.97 \%$ of within brain voxels possibly does not contain one WM trajectory. Third, most of them 
adopt fractional anisotropy (FA) as the stopping criteria. When FA is smaller than a certain value, fiber tracking process terminates making exploration of cortico-cortical connectivity impossible since FA near sub-cortical and cortical regions is very small. Therefore, these deterministic fiber tracking approaches work best on datasets with high FA and without voxel containing multiple WM trajectories. In addition, they cannot reveal sufficient and reliable information for analysis of human brain anatomical connectivity.

More recently, several probabilistic fiber tracking approaches 812 1314] have been proposed to address the three limitations to deterministic approaches by modelling uncertainty associated with PDD. Often, a probability density function (PDF) derived from either raw diffusion weighted images 814 or estimated diffusion tensor 1213 for each voxel, which estimates the probability of the orientation of local trajectories in all possible directions, is proposed. Then, simple line propagation is carried out using derived PDF of seed point as well as those voxels that the reconstructed trajectory passes through. This process is repeated in Monte-Carlo fashion to generate adequate number of WM trajectories to represent PDF of trajectories starting from seed point. Finally, statistical connectivity map is produced from reconstructed trajectories to estimate probability of connection from seed point to any other voxels. In 81314, such a map is estimated using Probabilistic Index of Connectivity (PICo), which is calculated as the number of trajectory passes through the region divided by the total number of trajectory reconstructed. This index is reasonable estimation of belief that there is a connection between two regions. And it is also adopted in this paper. Further normalization on this index is applied making comparison of connectivity starting from different regions feasible.

By studying previous probabilistic approaches, it was realized that there is still one problem in them, high curvature of reconstructed trajectories. It is believed that trajectory with low curvature means high belief of connection between two voxels. The factors that determine curvature of reconstructed $3 \mathrm{D}$ piecewise trajectory are turning angel (i.e. inverse cosine of dot product of previous direction and current direction vectors) and step size at each propagation step. In deterministic approach, low curvature is achieved by terminating fiber tracking process when turning angel is too high at the expense of coverage area of reconstructed trajectories. In probabilistic approach [812 13], such a priori information is not incorporated into PDF of local trajectory orientation. Therefore, most of reconstructed trajectories are irregular. In [14, Friman et al. considered the trajectory as first-order Markov Chain to minimize the probability of occurrence of large turning angel. However, step size was not taken into consideration. Furthermore, first-order Markov Chain is insufficient to minimize curvature, especially when trajectory passes through regions with low FA.

Inspired by work [14, a new probabilistic fiber tracking approach is presented in this paper. The approach models the trajectory as high order Markov Chain, which means that current direction is determined by several previous directions. At each propagation step, unlike previous approaches, both the current direction and step size are sampled. This new approach was applied to young normal DTI 
data to study trajectories passing through splenium of corpus callosum, then compared with [13].

\section{Method}

The reconstructed WM trajectory $x$ is a piecewise continuous $3 \mathrm{D}$ space curve which is considered as a sequence of space vector $\left\{x_{k}, k=1,2, \ldots, N\right\}$. The reconstruction process can be modelled as sequential sampling using equation (1)

$$
x_{k+1}=x_{k}+\alpha_{k} \nu_{k}
$$

where $\nu_{k}$ and $\alpha_{k}$ are the direction vector and step size of $k^{t h}$ sampling step, respectively.

WM trajectory $x$ is modelled as $m^{\text {th }}$ order Markov chain to regularize the reconstructed trajectory, that is, $\left(\nu_{k}, \alpha_{k}\right)$ depend on previous $m$ sampling steps. In this paper, Monte Carlo estimation of anatomical connectivity from region $\mathrm{A}$ to region $\mathrm{B} \theta_{A B}=E_{\pi} h_{A B}(x)$ is of interest, where $x$ is a random WM trajectory sampled from the distribution $\pi(x)$ can be expressed as:

$$
\begin{gathered}
\pi(x)=P\left(\nu_{1}, \alpha_{1} \mid y, \hat{D}\right) \prod_{l=2}^{m} P\left(\nu_{l}, \alpha_{l} \mid \nu_{l}, \ldots, \nu_{l-1}, y, \hat{D}\right) \\
\prod_{k=m+1}^{N} P\left(\nu_{k}, \alpha_{k} \mid \nu_{k-1}, \ldots, \nu_{k-m}, y, \hat{D}\right)
\end{gathered}
$$

where $\hat{D}$ is estimated diffusion tensor using multivariate linear regression, and $y$ is logarithm of raw diffusion weighted images (DWI).

In 814, posterior PDF of PDD $\nu_{k}$ and $\alpha_{k}$ is derived from DWI using twotensor model, which assumes two fiber trajectories with different orientation present within each voxel. Although problem of incomplete modelling in single tensor model is partially resolved, more data acquisitions as well as computation effort are required since two-tensor model involves more latent parameters. Considering limited acquisition time and limitations inherent to MR Imaging scanner (i.e. millimeter-scale resolution of DTI data), single tensor model is a reasonable compromise to reflect the averaged diffusion coefficient over a voxel in any direction of space. Therefore, posterior PDF of PDD $\nu_{k}$ and $\alpha_{k}$ is derived from estimated diffusion tensor $\hat{D}$. Any additional trajectory within a voxel is simply considered as uncertainty. And $\nu_{k}$ 's posterior distribution becomes disperser when multiple trajectories present within a voxel. Furthermore, instead of constant step size, it is adaptable so that the lower the uncertainty is, the larger it is.

The anatomical connectivity from region A to region B is measured using Probabilistic Index of Connectivity in 81314 by first sampling a large number of trajectories starting from region $\mathrm{A}$, and calculating the proportion of trajectories that pass through region $\mathrm{B}$. This measure indicates the belief that there 
is connection from region $\mathrm{A}$ to region $\mathrm{B}$. Note that this index is not symmetric. To compute connectivity from region $B$ to $A$, sampling trajectories starting from region $\mathrm{B}$ needs to be performed. This index of connectivity is not comparable suppose the starting region is different. Therefore, we proposed a coefficient associated with starting region, which measures the true relative number of trajectories passing through the region. And if the starting region is gray matter (GM), the volume of the region is a good candidate. Here, we assume the neurons are uniformly distributed in the region. If the starting region is WM, average FA is chosen. Currently, only comparison of connectivity starting from homogeneous brain tissue is possible. The connectivity function $h_{A B}(x)$ is:

$$
h_{A B}(x)=\left\{\begin{array}{cc}
c_{A} & \text { If x passes through region } \mathrm{B} \\
0 & \text { otherwise }
\end{array}\right.
$$

\subsection{Diffusion Tensor Estimation}

DT-MR Imaging consists of acquiring DWI $I_{i}, i=1,2, \ldots, K ; K \geq 6$, which measures a single scalar apparent diffusion constant (ADC) along different diffusionsensitizing directions $g_{i}, i=1,2, \ldots, K ; K \geq 6$. In DT-MR Imaging, diffusion tensor $D$ that characterizes anisotropic water diffusion within a macroscopic voxel is estimated from the set of at least 6 DWIs with non-collinear and noncoplanar diffusion-sensitizing directions, which are uniformly distributed on a unit sphere surface, plus the non-diffusion weighted image $I_{0}$ (i.e. $b=0$ ) using equation (4) [16 17] via multivariate linear regression (equation (5)):

$$
\begin{gathered}
I_{i}=I_{0} \exp \left(-b g_{i}^{T} D g_{i}\right) \\
Y=X \beta+\varepsilon, \varepsilon N\left(0, \sigma^{2}\right)
\end{gathered}
$$

where

$$
\begin{gathered}
Y_{i}=\ln I_{i}-\ln I_{0} \\
X=-b\left(\begin{array}{cccccc}
g_{1 x}^{2} & g_{1 y}^{2} & g_{1 z}^{2} & 2 g_{1 x} g_{1 y} & 2 g_{1 x} g_{1 z} & 2 g_{1 y} g_{1 z} \\
g_{2 x}^{2} & g_{2 y}^{2} & g_{2 z}^{2} & 2 g_{2 x} g_{2 y} & 2 g_{2 x} g_{2 z} & 2 g_{2 y} g_{2 z} \\
\vdots & \vdots & \vdots & \vdots & \vdots & \vdots \\
g_{K x}^{2} & g_{K y}^{2} & g_{K z}^{2} & 2 g_{K x} g_{K y} & 2 g_{K x} g_{K z} & 2 g_{K y} g_{K z}
\end{array}\right) \\
\beta=\left[\begin{array}{llllll}
D_{x x} & D_{y y} & D_{z z} & D_{x y} & D_{x z} & D_{y z}
\end{array}\right]^{T}
\end{gathered}
$$

where b-value renders the amount of diffusion weighting.

Then eigenvalues, eigenvectors, and FA were determined. A mask was also generated based on FA map to prevent fiber tracking outside brain. 


\subsection{Estimation of Joint Posterior Distribution}

This probability relates estimated diffusion tensor $\hat{D}$ to WM trajectory local direction $\nu_{k}$ and $\alpha_{k}$. Assume that $\nu_{k}$ and $\alpha_{k}$ are conditionally independent, the joint posterior distribution was decomposed into two distributions (equation (6) ). The key point is to introduce the a priori knowledge of low curvature of trajectories into posterior PDF. By applying Bayes' theorem, such a priori knowledge was incorporated in the form of prior distribution $P\left(\nu_{k} \mid \nu_{k-1}, \ldots, \nu_{k-m}\right)$ (equation (7))

$$
\begin{gathered}
P\left(\nu_{k}, \alpha_{k} \mid \nu_{k-1} \ldots \nu_{k-m}, Y, \hat{D}\right)= \\
P\left(\alpha_{k} \mid \nu_{k}, \nu_{k-1} \ldots \nu_{k-m}, Y, \hat{D}\right) \\
P\left(\nu_{k} \mid \nu_{k-1} \ldots \nu_{k-m}, Y, \hat{D}\right) \\
P\left(\nu_{k} \mid \nu_{k-1} \ldots \nu_{k-m}, Y, \hat{D}\right)=\frac{P\left(Y \mid \nu_{k} \ldots \nu_{k-m}, \hat{D}\right) P\left(\nu_{k} \mid \nu_{k-1} \ldots \nu_{k-m}, Y\right)}{P\left(Y \mid \nu_{k-1} \ldots \nu_{k-m}, \hat{D}\right)}
\end{gathered}
$$

Since single tensor model was used here, there are no other latent parameters besides estimated diffusion tensor $\hat{D}$. Assume Y depends on current propagation direction only; the equation (7) can be further simplified into:

$$
P\left(\nu_{k} \mid \nu_{k-1} \ldots \nu_{k-m}, Y, \hat{D}\right)=\frac{P\left(Y \mid \nu_{k}, \hat{D}\right) P\left(\nu_{k} \mid \nu_{k-1} \ldots \nu_{k-m}\right)}{\int_{\nu_{l}} P\left(Y \mid \nu_{l}, \hat{D}\right) P\left(\nu_{l} \mid \nu_{l-1} \ldots \nu_{l-m}\right)}
$$

It is impossible to evaluate integral term of equation (8) in continuous domain. Hence, $\nu_{k}$ is discretized into a large number of samples by uniformly sampling the sphere to transform integration into summation. The multivariate linear regression model assumes Gaussian distributed noise. The likelihood of $Y P\left(Y \mid \nu_{k}, \hat{D}\right)$ was modelled as:

$$
P\left(Y \mid \nu_{k}, \hat{D}\right)=\prod_{i=1}^{K} \frac{1}{\hat{\sigma} \sqrt{2 \pi}} \exp \left(-\frac{\left(Y_{i}+b g_{i}^{T} R\left(\nu_{k}\right) \hat{D} R\left(\nu_{k}\right)^{T} g_{i}\right)^{2}}{2 \hat{\sigma}^{2}}\right)
$$

where $R\left(\nu_{k}\right)$ is a rotation matrix that rotates PDD to $\nu_{k}$; the noise variance $\sigma^{2}$ was estimated using equation (10).

$$
\hat{\sigma}^{2}=\frac{\sum_{i=1}^{K}\left(Y_{i}+b g_{i}^{T} \hat{D} g_{i}\right)^{2}}{K-6}
$$

The prior distribution $P\left(\nu_{k} \mid \nu_{k-1} \ldots \nu_{k-m}\right)$ follows:

$$
P\left(\nu_{k} \mid \nu_{k-1} \ldots \nu_{k-m}\right) \propto\left\{\begin{array}{cc}
\left\langle\nu_{k}, \frac{x_{k}-x_{k-m}}{\left\|x_{k}-x_{k-m}\right\|}\right\rangle & \left\langle\nu_{k}, \frac{x_{k}-x_{k-m}}{\left\|x_{k}-x_{k-m}\right\|}\right\rangle>0 \\
0 & \text { otherwise }
\end{array}\right.
$$

This prior distribution gives the direction made up of low curvature trajectory high probability. In addition, the constraint in equation (11) is to avoid backward tracking. 
Distribution for step size $P\left(\alpha_{k} \mid \nu_{k}, \nu_{k-1} \ldots \nu_{k-m}, \hat{D}\right)$ follows Gaussian distribution with mean $c F A_{k}\left\langle\nu_{k}, \frac{x_{k}-x_{k-m}}{\left\|x_{k}-x_{k-m}\right\|}\right\rangle+b$ (where b and $\mathrm{c}$ are constant) and standard deviation $\sigma$. Therefore, when trajectory passes through regions with high FA and turning angle is small, the probability of $\alpha_{k}$ being large is high.

\subsection{Sampling and Re-sampling Trajectory}

The simple line propagation approach used for sampling trajectory is called Fiber Assignment by Continuous Tracking (FACT) described in our previous work [18. In brief, starting from user-defined seed voxel, fiber trajectory is reconstructed from the diffusion tensor by propagating forward and backward, following the PDD. As given in equation (11), suppose the current point is $x_{k}$, the next point $x_{k+1}$ along the path is calculated by adding the normalized PDD $\nu_{k}$ multiplied by the step size $\alpha_{k}$, where $\left(\nu_{k}, \alpha_{k}\right)$ is randomly generated using its joint posterior distribution. The tracking process is terminated when the net change in direction within a single voxel exceeded $\pm \pi / 2[13$, or the boundary of brain is reached. This is to compare our results with [13], since different stopping criterion may give different results. Note that, diffusion tensor $D$ is discrete, but continuous tensor field is required in propagation process. A statistical interpolation approach proposed by [8] is adopted to interpolate diffusion tensor field. In this statistical framework, diffusion tensor at point $x_{k}$ is assigned one of its nearest neighbors tensor values. The probability of picking one neighbor is inversely proportional to the distance between point $x_{k}$ and center of the neighbor. To estimate the connectivity from starting point to other regions, re-sampling of generated WM trajectories is performed to give Monte Carlo estimation as well as its standard error.

\section{Results}

The proposed probabilistic fiber tracking approach was carried out on real DTI data. DTI data consists of 15 DWIs and one non-diffusion weighted image. Images were obtained from one healthy volunteer on a Philips 3T MRI scanner using pulsed-gradient echo planar sequence with the following parameters: field of view $(\mathrm{FOV})=230 \mathrm{~mm} ; T R=3700 \mathrm{~ms} ; \mathrm{TE}=56 \mathrm{~ms} ; 256 \times 256$ acquisition matrix; slice thickness $=3 \mathrm{~mm}$; b factor $=800 \mathrm{smm}^{-2}$. All scans were approved by ethics committee of National Neuroscience Institute, Singapore.

To validate the results, the corpus callosum, which is the largest fiber bundle interconnecting the two cerebral hemispheres, was chosen to be studied, since its topography has been well defined in literature (e.g. 1920121]). The seed point (Fig.11) was placed on the midline in splenium (posterior part) of corpus callosum and 1000 trajectories were generated for first-order, third-order and fifth-order Markov Chain, respectively. We also defined a region of interest (ROI) (Fig. 1) composed of cuneus, superior occipital gyrus, middle occipital gyrus to study the anatomical connectivity from splenium of corpus callosum to occipital lobe. As suggested by previous qualitative and quantitative studies [14/192021, it 


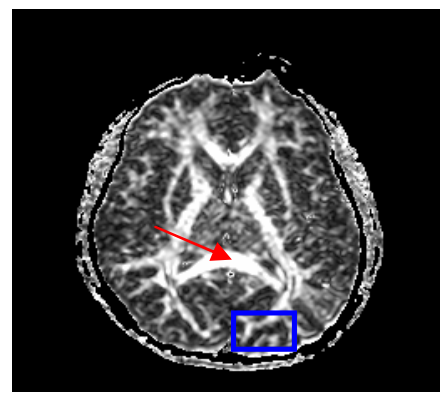

Fig. 1. Fractional Anisotropy map at middle axial slice. The red arrow shows the seed point; the blue rectangle shows region of interest.

is expected the connectivity is nearly 1 as most of fiber trajectories through splenium span out to occipital lobe, and a few, named tapetum, extend laterally on left side of human brain into the inferior temporal lobe.

In most previous probabilistic fiber tracking approaches, constant step size was used. And only direction vector was sampled at each propagation step. Compared to one of previous approach 13. (referred to as GJM method), the proposed method which incorporates adaptive step size gives less average number of sampling steps (table 1). Hence, the adaptive step size ensures effective sequential sampling of fiber trajectory. Furthermore, GJM method did not involve any a priori information of direction vector which controls the curvature of sampled fiber trajectories. Consequently, probability of violation of stopping criterion due to noise or partial volume effect before reaching ROI is higher than proposed method. In conclusion, a priori information helps reduce curvature of sampled fiber trajectories and fiber tracking process pass through noisy or branching regions. This is also confirmed by average curvature and connectivity shown in table [1. Since many sampled fiber trajectories by GJM method stops before they enter into ROI, the connectivity index is much lower than proposed method. In addition, high order Markov Chain does not necessarily give lower curvature. That curvature of sampled trajectories modelled by third and fifth order Markov Chain is higher than those modelled by first order Markov

Table 1. Comparison of characteristics of generated fiber trajectories by different method

\begin{tabular}{llll}
\hline Method & Step & Curvature & Connectivity \\
\hline GJM Method & $186.21 \pm 84.49$ & $0.1208 \pm 0.0914$ & $0.4807 \pm 0.0153$ \\
$1^{\text {st }}$ Markov Chain & $128.42 \pm 53.33$ & $0.1048 \pm 0.0429$ & $0.9074 \pm 0.0085$ \\
$3^{\text {rd }}$ Markov Chain & $148.33 \pm 64.15$ & $0.1148 \pm 0.0439$ & $0.9761 \pm 0.0049$ \\
$5^{\text {th }}$ Markov Chain & $148.93 \pm 60.57$ & $0.1127 \pm 0.0411$ & $0.9812 \pm 0.0044$ \\
\hline
\end{tabular}




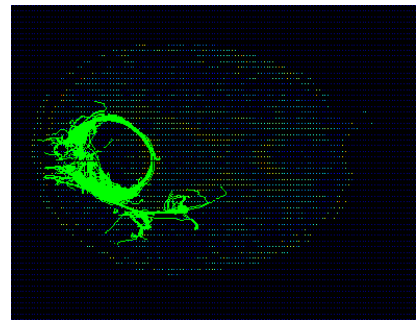

(a)

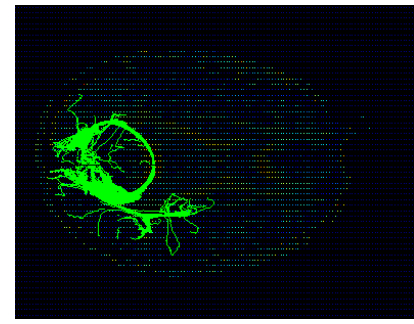

(b)

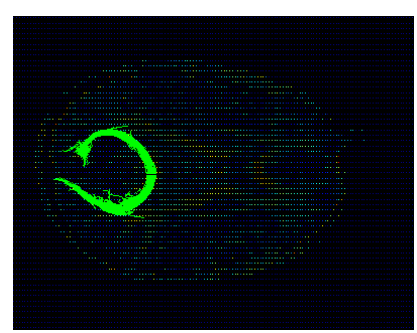

(c)

Fig. 2. Top view of generated fiber trajectories (a) 3rd order Markov Chain; (b) 5th order Markov Chain; (c) GJM Method

Chain, since high order Markov Chain makes violation of stopping criterion more difficult and trajectories near sub-cortical and cortical regions are more curved in nature.

Fig. 2 shows top view of sampled fiber trajectories by proposed method (Fig. 2](a) and (b) and GJM method (Fig. 2](c)]. Given same number of samples, the proposed method discovered more branches than GJM method. The proposed method found trajectories that project into inferior temporal lobe (i.e. tapetum), which were not found by GJM method. Hence, the convergence rate of PDF in proposed method is possibly faster than PDF in GJM method. And fewer samples are required to estimate the anatomical connectivity. In conclusion, the proposed method gives better estimation of PDF of fiber trajectory as well as anatomical connectivity.

Although high order Markov Chain is very effective in fiber tracking process, higher order does not mean better propagation results. The order of Markov Chain depends on the length of trajectory as well as its shape. If the trajectory is not so long and is very straight, lower order is sufficient to regularize the trajectory. Furthermore, if the trajectory is curved, high order may possibly distort its shape. To study fiber bundles (consist of thousands of trajectories), Monte-Carlo simulation with different order of Markov Chain can be carried out to reveal different levels of information. For fiber trajectories passing splenium of corpus callosum, 3 is an appropriate order. There is no significant difference of characteristics of sampled fiber trajectories between third and fifth order. 


\section{Conclusion}

A new probabilistic fiber tracking approach allowing effective regularization of reconstructed trajectories as well as exploration of cortico-cortical anatomical connectivity was proposed. The advantage of our approach over previous ones is that both turning angel and step size are taken into consideration to regularize reconstructed trajectories. Furthermore, there is no need to specialize threshold for FA or turning angel, which is usually difficult to determine, to terminate fiber tracking process. This helps fiber tracking process passes through noisy or branching regions and propagates into sub-cortical and cortical regions so that estimation of cortico-cortical anatomical connectivity is feasible.

Future work may include development of technique to fuse anatomical connectivity with function connectivity derived from fMRI.

\section{References}

1. Mori, S., van Zijl, P.C.M.: Fiber tracking: principles and strategies - a technical review. NMR Biomed. 15, 468-480 (2002)

2. Mori, S., Crain, B.J., Chacko, V.P., van Zijl, P.C.M.: Three dimensional tracking of axonal projections in the brain by magnetic resonance imaging. Ann. Neurol. 45, 265-269A (1999)

3. Xue, R., van Zijl, P.C.M., Crain, B.J., Solaiyappan, M., Mori, S.: In vivo threedimensional reconstruction of rat brain axonal projections by diffusion tesnor imaging. Magn. Reson. Med. 42, 1123-1127 (1999)

4. Jones, D.K., Simmons, A., Williams, S.C., Horsfield, M.A.: Non-invasive assessment of axonal fiber connectivity in the human brain via diffusion tensor MRI. Magn. Reson. Med. 42, 37-41 (1999)

5. Basser, P.J., Pajevic, S., Pierpaoli, C., Duda, J., Aldroubi, A.: In vivo fiber tractography using DT-MRI data. Magn. Reson. Med. 44, 625-632 (2000)

6. Lazar, M., Weinstein, D., Hasan, K., Alexander, A.L.: Axon tractography with tensorlines. In: Proceedings of the 8th Annual Meeting of ISMRM, Denver, pp. 482-482 (2000)

7. Conturo, T.E., Lori, N.F., Cull, T.S., Akbudak, E., Snyder, A.Z., Shimony, J.S., McKinstry, R.C., Burton, H., Raichle, M.E.: Tracking neuronal fiber pathways in the living human brain. Proc. Natl. Acad. Sci. USA 96, 10422-10427 (1999)

8. Behrens, T.E.J., Woolrich, M.W., Jenkinson, M., Johansen-Berg, H., Nunes, R.G., Clare, S., Matthews, P.M., Brady, J.M., Smith, S.M.: Characterization and Propagation of Uncertainty in Diffusion-Weighted MR Imaging. Magn. Reson. Med. 50, 1077-1088 (2003)

9. Makris, N., Worth, A.J., Sorensen, A.G., Papadimitriou, G.M., Wu, O., Reese, T.G., Wedeen, V., Davis, T., Stakes, J.W., Caviness, V.S., Kaplan, E., Rosen, B., Pandya, D.N., Kennedy, D.N.: Morphometry of in vivo human white matter association pathways with diffusion-weighted magnetic resonance imaging. Ann. Neurol. 42, 951-962 (1997)

10. Makris, N., Meyer, J.W., Bates, J.F., Yeterian, E.H., Kennedy, D.N., Caviness, V.S.: MRI-based topographic parcellation of human cerebral white matter and nuclei II. Rationale and applications with systematics of cerebral connectivity. Neuroimage 9, 18-45 (1999) 
11. Wiegell, M.R., Larsson, H.B.W., Wedeen, V.J.: Fiber crossing in human brain depicted with diffusion tensor MR imaging. Radiology 217, 897-903 (2000)

12. Hagmann, P., Thiran, J.P., Jonasson, L., Vandergheynst, P., Clarke, S., Maeder, P., Meuli, R.: DTI mapping of human brain connectivity: statistical fiber tracking and virtual dissection. NeuroImage 19, 545-554 (2003)

13. Parker, G.J.M., Haroon, H.A., Claudia, A.M.: Wheeler-Kingshott: A Framework for a Streamline-Based Probabilistic Index of Connectivity (PICo) Using a Structural Interpretation of MRI Diffusion Measurements. J. Magn. Reson. 18, 242-254 (2003)

14. Friman, O., Farneback, G., Westin, C.-F.: A Bayesian Approach for Stochastic White Matter Tractography. IEEE Trans. Med. Imag. 25, 965-978 (2006)

15. Mattiello, J., Basser, P.J., Lebihan, D.: Analytical expression for the b matrix in NMR diffusion imaging and spectroscopy. J. Magn. Reson. A 108, 131-141 (1994)

16. Basser, P.J., Mattiello, J., Lebihan, D.: Diagonal and off-diagonal components of the self-diffusion tensor: their relation to and estimation from the NMR spin-echo signal. In: 11th Annual Meeting of the SMRM, Berlin, p. 1222 (1992)

17. Basser, P.J., Mattiello, J., Lebihan, D.: Estimation of the effective self-diffusion tensor from the NMR spin-echo. J. Magn. Reson. B 103, 247-254 (1994)

18. Zheng, B., Rajapakse, J.C.: Effect of diffusion weighting and number of sensitizing directions on fiber tracking in DTI. Neural Information Processing, 102-109 (2006)

19. Parker, G.J.M., Wheeler-Kingshott, C.A.M., Barker, G.J.: Estimating Distributed Anatomical Connectivity Using Fast Marching Methods and Diffusion Tensor Imaging. IEEE Trans. on Med. Imag. 21, 505-512 (2002)

20. Hofer, S., Frahm, J.: Topography of the human corpus callosum revisitedComprehensive fiber tractography using diffusion tensor magnetic resonance imaging. NeuroImage 32, 989-994 (2006)

21. Jones, D.K., Griffin, L.D., Alexander, D.C., Catani, M., Horsfield, M.A., Howard, R., Williams, S.C.R.: Spatial Normalization and Averaging of Diffusion Tensor MRI Data Sets. NeuroImage 17, 592-617 (2002) 